\title{
Aceptación de la clase de educación física en escuelas privadas y públicas del Colima*
}

\author{
[Artículos]
}

\author{
Isela Guadalupe Ramos Carranza ${ }^{* *}$ \\ Adriana Isabel Andrade Sánchez ${ }^{* * *}$ \\ Rossana Tamara Medina Valencia ${ }^{* * * *}$ \\ Ciria Margarita Salazar C. ${ }^{* * * * *}$
}

Recibido: septiembre 19 del 2020

Aceptado: octubre 21 del 2020

Citar como:

Ramos Carranza, I. G., Andrade Sánchez, A. I., Medina Valencia, R. T. y Salazar C., C. M. (2021). Aceptación de la clase de educación física en escuelas privadas y públicas del Colima. Revista de Investigación Cuerpo, Cultura y Movimiento, 11(1). https://doi.org/10.15332/2422474X.6466

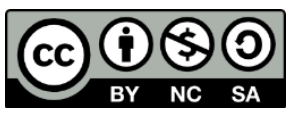

\footnotetext{
* Articulo resultado de investigación. Vinculado con el grupo de investigación Educación y movimiento UCOL-85. Colima, Colima, México.

** Doctora en Ciencias de la Cultura Física. Profesora e investigadora de tiempo completo. Universidad de Colima, México. Correo electrónico: iramos5@ucol.mx; ORCID: https://orcid.org/0000-0002-0629-9635

*** Doctora en Estadística Multivariante Aplicada. Profesora nivel superior y posgrado. Universidad de Colima, México. Correo electrónico: isa_andrade@ucol.mx; ORCID: https://orcid.org/0000-0001-9790-5719

**** Doctora en Educación Física y Artística. Profesora e investigadora de tiempo completo. Universidad de Colima, México. Correo electrónico:

rossanamedina@ucol.mx; ORCID: https://orcid.org/0000-0002-3664-9595

***** Doctora Educación Física y Artística. Profesora e investigadora de tiempo completo. Universidad de Colima, México. Correo electrónico: ciria6@ucol.mx; ORCID: https://orcid.org/0000-0001-8863-2309
} 


\section{Resumen}

El presente estudio tiene como objetivo identificar el nivel de aceptación que tienen los estudiantes de educación básica hacia la clase de educación física; además de conocer el grado de importancia que le otorgan a esta materia. Se aplicó el instrumento a 302 estudiantes de cuatro primarias de educación básica del estado de Colima, dos públicas y dos privadas. La edad de los estudiantes oscilaba entre los 8 y los 13 años con una media de 10.35 años ( \pm 0.93$)$, en los grados de $4^{\circ}, 5^{\circ}$ y $6^{\circ}$. Se utilizó el CAEF (Cuestionario de Actitudes hacia la Educación Física), que está estructurado en cuatro bloques: datos sociodemográficos, opinión sobre las clases de educación física, opinión sobre el profesorado de educación física, y hábitos de actividad física. Los resultados más relevantes arrojan que los estudiantes consideran la educación física como algo de poca utilidad ( $\mu=1.97 ; \pm 0.43)$; sin embargo, la valoración de la asignatura y del profesor de educación física es el factor con mayor puntaje de los siete analizados. Se puede concluir que la clase de educación física tiene un grado de aceptación elevado por los estudiantes; asimismo, los estudiantes del sector privado parecen valorar más la asignatura y al profesor encargado que los estudiantes de las escuelas públicas.

Palabras clave: educación física, actitudes, motivos, educación primaria

\section{Acceptance towards physical education class in private and public schools in Colima}

\section{Abstract}

The objective of this study is to identify the level of acceptance that basic education students have towards the physical education class; in addition to knowing the degree of importance they give to this subject. The instrument was applied to 302 students in four primary schools of basic education in the state of Colima, two public and two private. The 
students' age ranged from 8 to 13 years with an average of 10.35 years ( \pm o.93), in grades $4^{\text {th }}, 5^{\text {th }}$ and $6^{\text {th }}$. The CAEF (Questionnaire of Attitudes towards Physical Education) was used, which is structured in four blocks: socio-demographic data, opinion on physical education classes, opinion on physical education teachers, and physical activity habits. The most relevant results show that students consider physical education to be of little use ( $\mu=1.97 ; \pm 0.43$ ); however, the evaluation of the subject and of the physical education teacher is the factor with the highest score of the seven analyzed. It can be concluded that the physical education class has a high degree of acceptance by students; likewise, students in the private sector seem to value the subject and the teacher more than students in public schools.

Keywords: physical education, attitudes, motives, primary education.

\section{Introducción}

En los últimos años, se ha revalorizado la educación física como contenido capaz de establecer una formación integral de la persona a través de manifestaciones motrices; este cambio de perspectiva ha sido auspiciado por el incremento de profesionales vinculados con esta área de conocimiento (Moreno et ál., 2006).

La aceptación de la clase de educación física en la educación básica se considera una pieza clave para intervenir o incluir programas específicos que ayuden a resolver problemas detectados por los maestros de educación física, tales como: obesidad, sedentarismo, algunas enfermedades derivadas de esto, entre otros problemas de carácter social. En este sentido, Piéron et ál., (2007) observaron un estudio sobre el pensamiento, el proceso, el comportamiento y las actitudes en la clase de educación física en la secundaria donde analizaron a 60 estudiantes, enlistando los intereses de los niños, los valores que demostraban, percepciones del gusto y disgusto por la realización de las actividades, la motivación por parte del 
maestro y compañeros, y cuáles son sus hábitos de hidratación, higiene personal y desempeño en conjunto; esto se analizó por medio de cuestionarios de intereses y compromiso, lo que arrojó los siguientes resultados: un $46 \%$ participa, un $41 \%$ carece de motivación o gusto, ellos fueron llamados niños complicados, y, por último, el $13 \%$ no participa en la clase.

Por otro lado, Pastur (2010) argumentó que la conducta a modificar sobre el tiempo en el que se ejecutan las tareas y repercusiones del proceso enseñanza-aprendizaje en la educación física, sirve para encontrar nuevas acciones que nos ayuden a regular las conductas y modales de los alumnos, para tratar de dirigir estrategias y soluciones que disminuyan la impuntualidad, el retardo de los alumnos al llegar a clase y el porqué de la aptitud baja. Los resultados de esta parte se obtuvieron aplicando un cuestionario sobre las conductas de los niños con una muestra de 15 alumnos de 10 y 11 años (11 niños y 4 niñas), donde $47 \%$ de ellos llegan tarde por falta de interés.

La motivación en educación física es primordial, ya que se considera que es un sentir interno que te permite hacer algo; los estudios relacionados a esta investigación analizan como variable las propiedades psicométricas de un instrumento para valorar el tipo de regulación motivacional, que consta de saber de qué manera se debe motivar a los alumnos durante la clase. El método que señala dicha investigación se basa en un cuestionario de motivación en educación física, en donde se evalúa la satisfacción de las necesidades psicológicas del sujeto; esto determina el impacto motivacional del maestro y cómo este influye en la clase para que se cumplan los objetivos, metas y la intención pedagógica (Sánchez et ál., 2012).

El objetivo de esta investigación es identificar el nivel de aceptación que tienen los estudiantes de educación básica por la clase de educación física; 
además de conocer el grado de importancia que le otorgan a esta materia, con base en la variable de la motivación que el maestro transmite en la clase.

\section{Los motivos de participación}

La participación tiene un papel fundamental no solo en la familia, escuela y ámbito social, sino en el desarrollo dentro de diversos grupos. Esta herramienta puede facilitar la toma de decisiones y la libertad para actuar o decir las cosas que se crean correctas en el momento.

Lo anterior se refleja en el desempeño del estudiante en clase, porque dependiendo de qué tan motivado se sienta el niño estará de ánimo para realizar las actividades que el profesor le indique. La motivación, en palabras de Hampton et ál., (1989), está relacionada con todas las acciones que realiza una persona, es decir, que la motivación depende mucho de las decisiones que toma una persona para llevar a cabo una acción.

La participación es un medio en el cual la sociedad puede ser parte, tomar parte y sentirse parte de un grupo social. La aceptación de la educación física va más allá de ser una materia obligatoria y se convierte en el tiempo de disfrute y exploración social y emocional que un estudiante tiene durante su horario escolar.

\section{Gusto por la educación física}

La educación física es la materia en la cual los niños de una escuela aprenderán sobre la psicomotricidad de su cuerpo, trabajándola y conociéndola por medio de actividades que son principalmente juegos dentro de su grupo escolar; estas actividades también les ayudarán a tener una mejor socialización dentro de su grupo y una mejor convivencia. La SEP (2008), en su glosario de educación superior, menciona que la educación física es aquella en donde los alumnos deben conocer el 
desarrollo de sus cuerpos a través de actividades lúdicas que puedan ayudar al niño a mejorar el aspecto psicomotor. La educación física es brindada principalmente por la misma SEP, que debe ser la encargada de proporcionar este tipo de educación para el cuerpo o, en dado caso, se encargará el gobierno del estado en el cual está situada la institución que lo requiera. Ahora, viéndolo desde el punto de vista de Rodríguez (2008), la educación física es aquella donde existe un desarrollo corporal en el cual se trabajan hábitos, destrezas y movimientos que le ayudan al estudiante a adquirir capacidades físicas con un nivel técnico de habilidades que le permitan tener un buen desempeño dentro de las actividades que el docente ponga en práctica con los niños; además, ayuda a tener una formación integral en donde se vincula la satisfacción espiritual y la salud. El profesor juega un papel muy importante en la educación de los estudiantes, ya que es él quien va marcando las pautas a seguir para una educación de calidad que sea realmente útil para los niños; también es importante que el profesor brinde los mejores medios a los estudiantes, por medio de los cuales aprendan y contribuyan a su desarrollo escolar. Gadamer (2000) menciona que el educador, en este caso el profesor, es aquel que sirve como una guía para el estudiante dándole las herramientas necesarias para que este sea capaz de ser independiente al momento de tomar decisiones que él crea que sean las mejores para su desarrollo y que además sea responsable sobre si realmente está haciendo lo correcto al elegirlas (Beresaluce et ál., 2014).

Ahora, desde la perspectiva de la SEP (2008), el profesor tiene la labor de tomar las mejores decisiones para mejorar el aprendizaje de sus estudiantes. Este proceso de enseñanza-aprendizaje se va dando conforme el profesor observa cuáles son las herramientas más útiles con su grupo, a partir de la comprensión de que no todos los grupos y estudiantes

Revista de Investigación Cuerpo, Cultura y Movimiento ISSN: 2248-4418 | e-ISSN: 2422-474X | DOI: https://doi.org/10.15332/2422474X 
aprenden de la misma manera, ya que a algunos les funcionan métodos y técnicas que a otros no.

Romero (2009) menciona que el estudiante es aquel que es capaz de poseer el conocimiento de acuerdo con las herramientas que el profesor de clase le ha enseñado; asimismo, el niño será capaz de indagar por sí mismo qué le despierta la curiosidad por aprender más; de esta forma, podemos determinar que pondrá en práctica lo aprendido cuando lo requiera, utilizando la metodología que el docente ya le ha brindado con anterioridad.

\section{Jerarquía de las necesidades humanas}

Indiscutiblemente, al hablar de motivación es necesario hablar de Maslow (1943), puesto que es uno de los teóricos fundadores de la psicología humanística que postula que la sociedad busca la salud mental y para ello debe concretarse en la autorrealización de cada individuo. Maslow (1943) quería comprender el comportamiento que las personas tenían en los diferentes niveles de la pirámide de necesidades, así como la explicación de cómo la motivación jugaba un papel importante en la jerarquía señalada.

Se interpreta que en cada peldaño de su pirámide surgía una necesidad y comportamientos diferentes dentro de la vida del ser humano, que lo inducían a conductas negativas o positivas si estos niveles seguían su secuencia, que iban desde las necesidades fisiológicas hasta las necesidades de auto-relación. En esta última, se reforzaban las relaciones sociales y de estima. Si el ser humano era capaz de tener todos estos niveles equilibrados, obtendría una motivación significativa para entrar en un ámbito social importante (Maslow, 1943). 
Ahora, cada uno de estos niveles representa una parte importante en la vida del ser humano; no obstante, para esta investigación se abordará el tercer nivel que tiene que ver con lo social y representa el tercer grado de la pirámide que describe la amistad, las relaciones afectivas, la integración de los posibles clientes en un negocio, compañeros de escuela o trabajo y vecinos en una comunidad. Sentirse aceptado, pertenecer a un grupo o estado, logrará que las condiciones de vida del sujeto aumenten en cuanto a bienestar y sentimiento de competencia.

El profesor de educación física ayuda con la formación social y emocional del estudiante, mediante actividades que incentivan la cooperación y son llamativas para crear relaciones afectivas entre ellos; juegos como la red humana y cruzando la frontera, donde se pone en práctica el trabajo en equipo y que son actividades de comunicación-cooperación, son ejemplos de lo mencionado.

En la clase de educación física se ve en todo momento la autorrealización, ya que el profesor pone actividades de menor grado de dificultad a mayor; con ello se busca, primero, que el alumno experimente las demás etapas de la pirámide para que cuando lleguen a esta última sean capaces de ser creativos, dinámicos sin miedo a burlas y que puedan realizar las actividades más difíciles; con esto, las demás etapas deberán verse reflejadas para cumplir la pirámide de la motivación de Maslow.

Maslow (1943) dice que las necesidades deben cumplirse para que la pirámide tenga ese carácter de satisfacción del individuo; es importante llegar a la cima del esquema para que, en primera instancia, logren reconocer errores, resolver problemas, se autorrealicen y satisfagan cada uno de los niveles mencionados (Santrock, 2006).

Como lo dice la teoría sobre la educación física, se especifica que han ocurrido transformaciones en los avances sobre las disciplinas donde el 
pensamiento es concebido de manera que se relacione el cuerpo con el contexto educacional y físico, aportando nuevas ideas, conceptos y corrientes como lo son: la socio-motricidad, la investigación, la educación física como base de formación personal y de la psicomotricidad para formar deportistas (Pareja, 2010).

\section{Metodología}

Se trata de un estudio transversal con un alcance explicativo de tipo no experimental y de carácter descriptivo, el cual selecciona una serie de cuestiones, conceptos o variables y mide cada una de ellas independientemente de las otras, con el fin, precisamente, de describirlas (Hernández et ál., 2010). La técnica que se utilizó para la recolección de información fue el CAEF (Cuestionario de Actitudes hacia la Educación Física), que está estructurado en cuatro bloques: datos sociodemográficos, opinión sobre las clases de educación física, opinión sobre el profesorado de educación física y hábitos de actividad física. Consta de 56 ítems donde se les pregunta a los estudiantes el grado de conformidad con la clase de educación física; las respuestas van en una escala de tipo Likert donde parten desde el número 1 (Desacuerdo) hasta el 4 (Totalmente de acuerdo), dicho instrumento está validado y reforzado por el Instituto de Ciencias del Deporte de la Universidad del Sarre de Alemania (Cárcamo, 2012; Wydra, 2001), utilizando el método de back-traslation o método de traducción inversa, (Brislin, 1980) por su traducción al español.

Las respuestas al CAEF pueden agruparse en siete variables latentes que se conforman de la siguiente forma:

Tabla 1. Ítems que conforman las variables latentes o factores del CAEF

\begin{tabular}{|l|c|}
\multicolumn{1}{|c|}{ Variable latente } & \multicolumn{1}{c}{ Ítems } \\
\hline $\begin{array}{l}\text { Valoración de la asignatura y del profesor de } \\
\text { educación física }\end{array}$ & $6,10,17,18,21,30,37,38,43,53$ y 55 \\
\hline
\end{tabular}

Revista de Investigación Cuerpo, Cultura y Movimiento

ISSN: 2248-4418 | e-ISSN: 2422-474X | DOI: https://doi.org/10.15332/2422474X

Vol. 11 N. 01 | enero-junio de 2021 


\begin{tabular}{|c|c|}
\hline Variable latente & Ítems \\
\hline Dificultad de la educación física & $4,11,19,26,36$ у 44 \\
\hline Utilidad de la educación física & $1,5,12,15,16,31,32,35,40$ y 42 \\
\hline Empatía con el profesor y la asignatura & $2,3,7,8,23$ y 48 \\
\hline $\begin{array}{l}\text { Concordancia con la organización de la } \\
\text { asignatura }\end{array}$ & $13,14,24,52$ y 54 \\
\hline Preferencia por la educación física y el deporte & $20,28,34$ y 50 \\
\hline La educación física como deporte & $25,45,47$ y 49 \\
\hline
\end{tabular}

Fuente: adaptado de: Moreno, J. A. et ál. (2003).

\section{Participantes}

Los participantes se seleccionaron por medio de un método de tipo no probabilístico, se trabajó con 302 estudiantes de cuatro primarias de educación básica del estado de Colima, dos públicas y dos privadas. La edad de los estudiantes oscilaba entre los 8 y los 13 años con una media 10.35 años ( \pm o.93), en los grados de $4^{\circ}, 5^{\circ}$ y $6^{\circ}$.

\section{Análisis de los datos}

El análisis de datos se realizó en el programa estadístico SPSS v.21. La descripción de los resultados se llevó a cabo a través de medidas de tendencia central (media) y dispersión (desviación típica). Para determinar si existen diferencias estadísticas según el tipo de escuela y sexo se analizaron las siete escalas del CAEF a través de la prueba $\mathrm{T}$ de Student, y para el análisis de acuerdo con el nivel educativo se utilizó una prueba de Anova. En todos los casos, el nivel de confianza fue del $95 \%$ $(\alpha=0.05)$.

\section{Resultados}

Como se señaló anteriormente, se trabajó con 302 estudiantes de cuatro primarias de educación básica del estado de Colima; 181 (59.9 \%) 
pertenecían al sector público y 121 (40.1\%) al privado; 153 de los estudiantes (50.7 \%) eran hombres y 149 (49.3 \%) mujeres. El promedio de edad de los alumnos fue de 10.35 años $( \pm 0.93)$ distribuidos en cuarto grado (74 alumnos, $24.5 \%$ ), quinto grado (107 estudiantes, $35.4 \%$ ), y sexto grado (121 alumnos, $40.1 \%$ ).

Además de las preguntas que integran el CAEF, se incluyeron una serie de preguntas sobre los hábitos en la práctica deportiva. De estos se puede destacar que el $90.4 \%$ tomaban clase por la mañana y solo un $9.6 \%$ por la tarde, mientras que el $99.1 \%$ de los grupos son mixtos. En lo que refiere a prácticas extraescolares, un $70.5 \%$ de los niños no tienen prácticas extraescolares y solo un $\mathbf{2 9 . 5} \%$ tienen alguna actividad fuera de la escuela primaria. Este porcentaje es muy similar al $34.4 \%$ de los padres de familia que practican algún tipo de deporte.

De los siete factores que conforman el CAEF, la utilidad de la educación física es el apartado con menor puntuación ( $\mu=1.97 ; \pm 0.43$ ), seguido de la dificultad ( $\mu=2.49 ; \pm 0.66)$, aunque es importante recordar que los ítems que conforman este factor se centran en la facilidad de ejecución de las actividades (p.e. "pregunta 4: las actividades de EF son fáciles"). La parte de valoración de la asignatura y del profesor de educación física es el factor con mayor puntaje de los siete analizados (tabla 2.)

En la búsqueda de diferencias significativas entre las puntuaciones otorgadas al CAEF y el sexo de los alumnos, se realizó la prueba de T de Student comparando los promedios de puntajes de ambos sexos. Considerando un p-valores resultantes con un nivel de confianza en el $95 \%$, es posible identificar que ninguna de las siete categorías tiene diferencias estadísticamente significativas. Es decir, las percepciones de los estudiantes hacia la educación física son independientes de su sexo (ver tabla 3).

Revista de Investigación Cuerpo, Cultura y Movimiento ISSN: 2248-4418 | e-ISSN: 2422-474X | DOI: https://doi.org/10.15332/2422474X Vol. 11 N. 01 | enero-junio de 2021 
Tabla 2. Puntuaciones medias a las categorías que conforman el CAEF

\begin{tabular}{|l|l|l|}
\hline & \multicolumn{1}{|c|}{$\boldsymbol{\mu}$} & $\mathbf{( \pm D . E . )}$ \\
\hline Valoración de la asignatura y del profesor de Educación física & 2.94 & 0.68 \\
\hline Dificultad de la educación física & 2.49 & 0.66 \\
\hline Utilidad de la educación física & 1.97 & 0.43 \\
\hline Empatía con el profesor y la asignatura & 2.45 & 0.66 \\
\hline Concordancia con la organización de la asignatura & 2.68 & 0.68 \\
\hline Preferencia por la educación física y el deporte & 2.52 & 0.73 \\
\hline La educación física como deporte & 2.30 & 0.73 \\
\hline
\end{tabular}

Fuente: elaboración propia.

Tabla 3. Puntuaciones medias a las categorías que conforman el CAEF según el sexo de los estudiantes

\begin{tabular}{|c|c|c|c|c|c|c|c|}
\hline & \multicolumn{3}{|c|}{ Hombres } & \multicolumn{3}{|c|}{ Mujeres } & \multirow[t]{2}{*}{ P-valor } \\
\hline & $\mathbf{N}$ & $\mu$ & ( \pm D.E.) & $\mathbf{N}$ & $\boldsymbol{\mu}$ & ( \pm D.E.) & \\
\hline Valoración & 153 & 2.91 & (o.68) & 149 & 2.96 & (o.68) & 0.554 \\
\hline Dificultad & 153 & 2.49 & (o.66) & 149 & 2.49 & (0.65) & 0.998 \\
\hline Utilidad & 153 & 2.01 & $(0.46)$ & 149 & 1.94 & (0.39) & 0.166 \\
\hline Empatía & 153 & 2.52 & (o.62) & 149 & 2.38 & (o.69) & 0.074 \\
\hline Concordancia & 153 & 2.75 & (o.68) & 149 & 2.61 & (o.68) & 0.066 \\
\hline Preferencia & 153 & 2.54 & (0.79) & 149 & 2.50 & $(0.67)$ & 0.616 \\
\hline La educación física como deporte & 153 & 2.29 & $(0.78)$ & 149 & 2.31 & (o.68) & 0.831 \\
\hline
\end{tabular}

Fuente: elaboración propia.

Uno de los objetivos de esta investigación fue conocer si existían actitudes distintas hacia la educación física dependiendo del sector educativo en el que se encontraban los alumnos. Para esto, se realizó una prueba de T de Student y se analizaron las respuestas de los estudiantes al CAEF, que pueden observarse en la tabla 4. Es importante resaltar que en seis de los siete factores que mide el cuestionario no se encontraron diferencias significativas entre los sectores, sin embargo, los estudiantes del sector privado parecen valorar más la asignatura y al profesor que la dirige $(\mu=$ $3.09 ; \pm 0.57)$ en comparación con el sector público $(\mu=2.83 ; \pm 0.73)$; de 
esta forma, se encontraron pruebas estadísticas suficientes (p-valor altamente significativo $=0.001$ ) para realizar esta afirmación.

Tabla 4. Puntuaciones medias a las categorías que conforman el CAEF según el sector educativo

\begin{tabular}{|l|c|c|c|c|c|c|c|}
\hline & \multicolumn{4}{|c|}{ Pública } & \multicolumn{4}{c|}{ Privada } & P-valor \\
\cline { 1 - 8 } & $\mathbf{N}$ & $\boldsymbol{\mu}$ & $\mathbf{( \pm \mathbf { D . E } . )}$ & $\mathbf{N}$ & $\mu$ & $\mathbf{( \pm D . E . )}$ \\
\hline Valoración & 181 & 2.83 & 0.73 & 121 & 3.09 & 0.57 & 0.001 \\
\hline Dificultad & 181 & 2.44 & 0.66 & 121 & 2.57 & 0.65 & 0.083 \\
\hline Utilidad & 181 & 2.01 & 0.47 & 121 & 1.92 & 0.35 & 0.083 \\
\hline Empatía & 181 & 2.49 & 0.67 & 121 & 2.39 & 0.62 & 0.201 \\
\hline Concordancia & 181 & 2.63 & 0.72 & 121 & 2.76 & 0.61 & 0.102 \\
\hline Preferencia & 181 & 2.55 & 0.75 & 121 & 2.49 & 0.69 & 0.464 \\
\hline La educación física como deporte & 181 & 2.32 & 0.75 & 121 & 2.27 & 0.69 & 0.550 \\
\hline
\end{tabular}

Fuente: elaboración propia.

Finalmente, se realizó el análisis en búsqueda de diferencias estadísticas entre las percepciones hacia la educación física y el año que cursaban los estudiantes. Para realizar las comparaciones según el grado escolar se llevó a cabo un Anova y los resultados obtenidos se presentan de forma detallada en la tabla 5 . Al observar detalladamente es posible resaltar como la dificultad de la educación física es el factor que muestra diferencias estadísticamente significativas entre los alumnos. Al realizar la prueba de Tuckey se encontró que esta diferencia se encuentra entre los alumnos de $4^{\circ}$ y el resto de los alumnos ( $\left.\mathrm{p}<0.05\right)$, ya que los niños de este grado escolar consideran tener más dificultades con la materia que el resto de los estudiantes. Es importante recordar al lector que los ítems que conforman este factor se centran la facilidad de la materia (p. E. "afirmación 11: Aprobar en E.F. es más fácil que en otras asignaturas"). 
Tabla 5. Puntuaciones medias a las categorías que conforman el CAEF según el grado escolar

\begin{tabular}{|c|c|c|c|c|c|c|c|c|c|c|}
\hline & \multicolumn{3}{|c|}{ Cuarto } & \multicolumn{3}{|c|}{ Quinto } & \multicolumn{3}{|c|}{ Sexto } & \multirow{2}{*}{$\begin{array}{c}\text { P- } \\
\text { valor }\end{array}$} \\
\hline & $\mathbf{N}$ & $\mu$ & ( \pm D.E.) & $\mathbf{N}$ & $\mu$ & ( \pm D.E.) & $\mathbf{N}$ & $\mu$ & ( \pm D.E.) & \\
\hline Valoración & 74 & 2.83 & 0.84 & 107 & 3.00 & 0.61 & 121 & 2.95 & 0.63 & 0.246 \\
\hline Dificultad & 74 & 2.31 & 0.65 & 107 & 2.56 & 0.67 & 121 & 2.55 & 0.63 & 0.023 \\
\hline Utilidad & 74 & 1.96 & 0.41 & 107 & 2.00 & 0.46 & 121 & 1.97 & 0.42 & 0.796 \\
\hline Empatía & 74 & 2.46 & 0.64 & 107 & 2.42 & 0.68 & 121 & 2.48 & 0.65 & 0.813 \\
\hline Concordancia & 74 & 2.58 & 0.71 & 107 & 2.70 & 0.58 & 121 & 2.72 & 0.73 & 0.315 \\
\hline Preferencia & 74 & 2.40 & 0.82 & 107 & 2.52 & 0.68 & 121 & 2.60 & 0.71 & 0.180 \\
\hline $\begin{array}{l}\text { La educación } \\
\text { física como } \\
\text { deporte }\end{array}$ & 74 & 2.33 & 0.78 & 107 & 2.30 & 0.76 & 121 & 2.28 & 0.67 & 0.917 \\
\hline
\end{tabular}

Fuente: elaboración propia.

\section{Conclusiones}

Los resultados obtenidos permiten concluir que la motivación y aceptación por la clase de educación física de acuerdo con la percepción de la escuela pública es de un $56.45 \%$, mientras que en el sector privado es de un 43.55 \%; que va desde la motivación brindada por el docente y que se mantiene durante la clase. De acuerdo con Sánchez et ál., (2012), la percepción se asemeja en ambos contextos en que los alumnos se sienten motivados si el maestro dirige bien la sesión y si se cumplen los objetivos.

Partiendo de la motivación y gusto por la clase de educación física, se puede señalar que los estudiantes que no participan en una clase de educación física no lo hacen por falta de motivación del profesor en el desarrollo de la clase; no obstante, es la clase favorita de los estudiantes de educación básica. Es decir, la aceptación por la clase actúa directamente en los estudiantes, independientemente del maestro y de la clase en sí misma. En definitiva, la influencia de los métodos, estrategias y formas de enseñanza de la educación física, se traducen en los intereses y motivación 
que puede transmitir el maestro durante una clase. En cuanto a las diferencias de sexo, se puede concluir que las mujeres prefieren juegos cooperativos y los hombres actividades que exijan pruebas de condición física, que midan sus habilidades deportivas; sin embargo, estadísticamente no existen diferencias significativas en cuanto al sexo. Además, la clase de educación física tiene un grado de aceptación elevado por parte de los estudiantes. Asimismo, los estudiantes del sector privado parecen valorar más la asignatura y al profesor de educación física en comparación con el sector público, encontrándose pruebas estadísticas suficientes para realizar esta afirmación.

Finalmente, podemos destacar que la aceptación de la clase de educación física es buena, centrándose en el valor que le otorgan a la materia. De esta forma queda en manifiesto que el maestro puede determinar y fomentar un estilo de vida saludable fuera de la escuela; el profesor es el responsable, en gran medida, de los futuros hábitos del niño, ahí radica la relevancia de establecer este tipo de investigación para que las estancias educativas establezcan programas adecuados para incrementar el gusto por la educación física, considerando los beneficios sociales y emocionales que le otorgan a los estudiantes.

\section{Referencias}

Díez, M. D. R. B., Peiró-i-Gregori, S., \& Hernando, C. R. (2014). El profesor como guíaorientador: Un modelo docente. En El reconocimiento docente. Innovar e investigar con criterios de calidad: XII Jornadas de redes de investigación en docencia universitaria (pp. 857-870). Instituto de Ciencias de la Educación.

Brislin, R. W. (1980). Translation and content analysis of oral and written material. En H. C. Triandis y J. W. Berry (Eds.), Handbook of cross-cultural psychology (389444). Allyn and Bacon.

Revista de Investigación Cuerpo, Cultura y Movimiento 
Cárcamo, J. (2012). El profesor de Educación Física desde la perspectiva de los escolares. Estudios pedagógicos, 38(1), 105-119. https://doi.org/10.4067/s0718$\underline{07052012000100006}$

Gadamer, H.G. (2000). Educación es educarse. Paidós.

Hampton, D., Summer, C. y Webber, R. (1989). Manual de desarrollo de recursos humanos. Trillas.

Hernández, R., Fernández, C. y Baptista. (2010). Metodología de la Investigación. Mcgraw Hill Interamericana.

Hernández, M. R. (2008). La actualidad de la educación física en el desarrollo integral del ser humano. InterSedes: Revista de las Sedes Regionales, 9(17), 121-133. https://www.redalyc.org/pdf/666/66615066009.pdf.

Maslow. (1943). Teoría de la Motivación y Jerarquía de las Necesidades de Maslow. Psychological Review, 50 (4), 370-396. https://psycnet.apa.org/doiLanding?doi=10.1037\%2Fho054346

Moreno, J. A., Martínez, C. y Alonso, N. (2006). Actitudes hacia la práctica físicodeportiva según el sexo del practicante. Revista Internacional de Ciencias del Deporte, 2(2), 20-43. https://doi.org/10.5232/ricyde2006.00302

Moreno, J. A.; Rodríguez, P. L. y Gutiérrez, M. (2003). Intereses y actitudes hacia la Educación Física. Revista española de educación física, 11(2), 14-28. http://gicom.umh.es/escalas/CAEF.pdf

Pareja, I. D. (2010). Teoría y práctica de la Educación Física. Expomotricidad.https://revistas.udea.edu.co/index.php/expomotricidad/a rticle/view/335267/20790932

Pastur, R. P. (2010). Modificación de la conducta de una clase de educación física: Utilización de estrategias psicológicas y pedagógicas para disminuir el tiempo de permanecia en vestuarios. Emásf, Revista Digital de Educación Física, 1 (5), 2035. https://doi.org/10.20511/usil.thesis/9306

Piéron, M., González, M. y Castro, M. (2006). Actitudes y Motivación en Educación Física. 10. Retos. Nuevas Tendencias en E.F. Deporte y Recreación. 
Romero, G. (2009). Aprendizaje significativo y constructivismo. Temas para la educación, Revista digital para los profesionales de enseñanza, 1(3), 1-8.

https://www.feandalucia.ccoo.es/docu/p5sd4981.pdf.

Sánchez, D., Leo, F., Amado, D., González, I. y García, T. (2012). Desarrollo de un Cuestionario para Valorar la Motivación en Educación Física. Revista iberoamericana de Psicología del Ejercicio y el Deporte, 7 (2), 227-250. https://doi.org/10.4321/s1578-84232015000300002

Santrock, J. (2006). Psicología de la Educación. McGraw Hill Interamericana.

Wydra, G. (2001). Beliebtheit und Akzeptanz des Sportunterrichts. Sportunterricht, 5O(3), 67-72. https://docplayer.org/29348304-Georg-wydra-2001-beliebtheitund-akzeptanz-des-sportunterrichts-sportunterricht-50-67-72.html 